CORRIGENDUM

\title{
Age-related patterns in mental health-related complementary and alternative medicine utilization in Canada
}

Rebecca Crabb and John Hunsley

doi:10.1017/S1041610210001328, Published by Cambridge University Press, 18 August 2010

The authors omitted the following statement from the Acknowledgments section: "The views expressed in this paper are those of the authors and not necessarily those of the Department of Veterans Affairs". 